\title{
A mini-module with embedded spacers for high-throughput ultrafiltration
}

\author{
Kristina Baitalow ${ }^{\mathrm{a}}$, Denis Wypysek ${ }^{\mathrm{a}, \mathrm{b}}$, Martin Leuthold ${ }^{\mathrm{c}}$, Stefan Weisshaar ${ }^{\mathrm{c}}$, Jonas \\ 4 \\ Lölsberg ${ }^{\mathrm{a}, \mathrm{b}}$, Matthias Wesslinga,b,* \\ ${ }^{a}$ RWTH Aachen University, Chemical Process Engineering, Forckenbeckstrasse 51, 52074 Aachen, Germany \\ ${ }^{b}$ DWI-Leibniz - Institute for Interactive Materials, Forckenbeckstrasse 50, 52074 Aachen, Germany \\ ${ }^{c}$ Sartorius Stedim Biotech GmbH, August-Spindler-Str. 11, 37079 Göttingen, Germany
}

\section{Abstract}

Ultrafiltration membrane modules suffer from performance losses that arise during filtration from concentration polarization and fouling. Such performance losses are frequently mitigated by controlling the hydrodynamic conditions at the membrane/fluid interface. For instance, the hydrodynamic conditions are manipulated using mesh spacers that act as a static mixer. The design of such spacers is rarely optimized to effectively maintain mass transport through the membrane. Also, the spacer is an additional part added to the feed channel of the membrane module, improving mass transport in general, yet accepting less transport in dead zones.

Here, we present a mini module with spacers embedded in the module housing of a flat-sheet ultrafiltration membrane to attain high permeation rates. The performance of two new embedded spacer geometries - staggered herringbone and sinusoidal corrugation - prove experimentally that indeed a CFD-simulated flux increase can be realized during bovine serum albumin (BSA) filtration. The flow characteristics inside the mini module are further investigated using magnetic resonance velocity imaging. The new embedded sinusoidal corrugation spacers outperform conventional mesh spacer inlays. The fabrication of such module-embedded spacers has been conceptually implemented through an in-silico design and a 3D-printing production process. The latter can be easily realized using injection molding processes, as is now done for the Sartorius ambr ${ }^{\circledR}$ crossflow product line.

Keywords: Ultrafiltration membrane module, embedded static mixers, fouling mitigation, CFD, Magnetic

Resonance Imaging

\section{Introduction}

pharmaceutical industries [1-3]. Ultrafiltration membranes remove harmful matter such as

bacteria and viruses, concentrate a desired product such as proteins and enzymes or separate

other valuable products from one another to increase purity [4].

Particulate and colloidal matter is omnipresent in biotechnological processes and tends to accumulate at the membrane surface, forming a complex deposit film on top of the

\footnotetext{
*Corresponding author: manuscripts.cvt@avt.rwth-aachen.de
} 
[6]. Matthiasson and Sivik have already stated in the 1980s that concentration polarization occurring in ultrafiltration processes leads to mass transfer inhibition [7]. To address this issue, various measures are taken, one of which are static mixers inserted into membrane modules to increase mixing and subsequently mass transfer [8]. Static mixers increase the shear rate and thus reduce fouling [9] induced by biological or colloidal matter. The mixers function as obstacles disrupting the laminar flow profiles and causing vortices in the flow field normal and away from the membrane surface. This induced mixing also leads to higher mesh type spacers show better mixing (high shear rate, low drag coefficient), but also an increase in pressure drop [10]. This leads to a constant trade-off between flux increase and the correlated rise in energy input due to higher pressure loss [11, 12].

The downside of mesh type spacers is that they require several assembling steps in line production. A single-step production such as injection molding could accelerate the production process and reduce operating costs. Removing such mesh spacers from a module architecture has been addressed by incorporating spacers as turbulence promoters into the membrane as so-called membrane-con-spacer geometries [13-15].

Studies on net- or diamond-shaped spacers and meshes are available in large numbers 42 and are discussed in several reviews $[12,16,17]$. The literature on experimental and simulation-based investigations of mesh spacers proves reduced biofouling and prolonged stable membrane-module performance [11, 18-20]. To describe such mesh spacers or static mixers, conventional literature uses simple 2D structures like triangles, squares or circles and 46 focuses on their position and dimensions [21, 22]. As those static mixers are not suitable for injection molding, they are not further discussed here.

48 Only few studies focus on module-embedded instead of inlay static mixers. A patterned membrane surface acts as spacer structure and induces vortices [23]. An implementation of commercial mesh spacers used as embedded spacer structure exists only for simple geometries such as squares [24]. A ladder-type spacer within a frame fabricated by 3D printing was studied in ultrafiltration experiments [25]. Here, the spacer elements direct the fluid flow to the upper and lower parts of the membrane module but contain undercuts, which is not 
54

suitable for injection molding. Other experiments show positive effects only in the entrance region for mesh type spacers and embedded ribbon-type spacers [26]. Another spacer type suitable for ultrafiltration is a zigzag spacer without any obstacles, which outperforms mesh-type spacers in terms of pressure drop [27]. Staggered herringbone structures are a good example for embedded spacer structures, but are suitable primarily for microfluidic applications [28]. Shrivastava et al. [29] examined asymmetric herringbone, helix and laddertype structures intending to provide a guide for developing reasonable spacers. Positive effects in mass transfer were found to be highest for helices, followed by herringbones and ladder-type spacers [29].

While much work deals with flow distribution only, some studies also address the corresponding simultaneous mass transfer phenomena quantitatively [11, 30? , 31]. Mass transfer enhancement is accompanied by an increase in axial pressure drop [31]. This results in an increase in mass transfer coefficients with increasing shear rates [30]. Mass transfer investigations were considered for simple geometries only [30,31], but recently our group has addressed membranes with spacers $[13,32,33]$ and 3D printed novel spacer and mixer geometries [34-36]. A general overview is given by the Virtual Issue of the Journal [37].

Only few studies investigate 3D printing-aided membrane modules, despite the great potential of 3D printing for fast and cheap module improvement [38]. Module design and material selection must be considered in 3D printing, but research on 3D printing of membrane process-related questions progresses rather slow [17].

Most of the research has so far focused on fluid flow simulations, simple 2D structures or inlay mesh-type spacers, which are not suitable for single-step production. 3D simulations are preferable to 2D simulations because the latter underestimate the parameters examined [18]. In this study, we therefore tackle 3D simulations including mass transfer, implement an obstacle-free spacer structure for a high-throughput membrane module and use 3D printing for fast prototyping. For the first time, we complement CFD simulations with both experimental validation and flow-MRI measurements. 


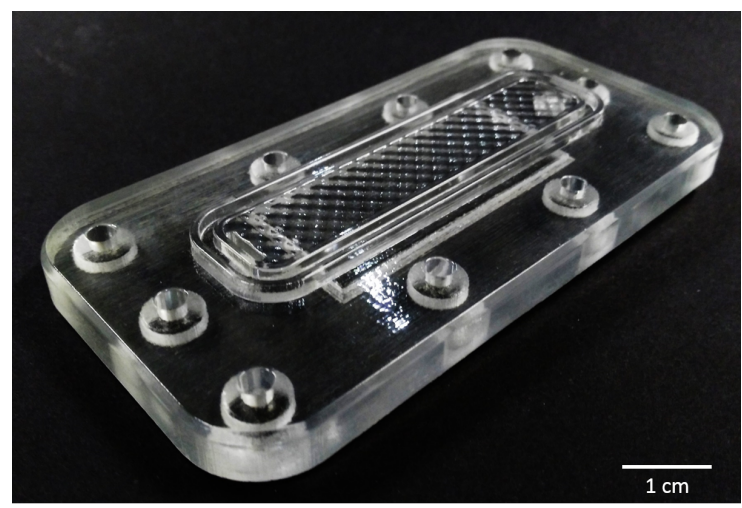

Figure 1: 3D printed membrane module with embedded sinusoidal spacers.

\section{Materials and methods}

\subsection{Additive manufactured membrane modules}

The mass transport properties for different embedded spacer geometries were evaluated in an additively manufactured membrane module that was fabricated using polyjet 3D printing (Stratasys, Objet Eden 260V). The membrane module shown in Figure 1 was printed layer by layer with a transparent photo-polymer (Stratasys, RGD810). During printing, the internal fluid channels need supporting structures (Stratasys, SUP705), which were later removed using a high-pressure washer (Krumm-tec, RK Top 5). Any remaining material was subsequently dissolved in a stirred bath of $1 \mathrm{~mol} \mathrm{~L}^{-1}$ sodium hydroxide for approximately 24 hours.

\subsection{Design of the embedded spacer}

The challenge is to design the membrane module for injection molding. The module investigated in this work is a small-scale cross flow module consisting of two parts. The first part contains the feed inlet and a retentate outlet. The feed inlet leads to a rectangular channel upon which the membrane is placed. The corners of the feed channel are rounded off in order to minimize material deposition in dead zones. The second part of the module contains a permeate outlet.

The advantage of replacing mesh spacers with embedded spacers is the possibility of single-step production that requires less time, e.g. injection molding without the need for a 
spacer placement step. During injection molding, polymer granulate is molten and transferred into a metal shape where it is injected into a mold cavity via a nozzle. For the design of the spacer embedded in the membrane module, any ramifications or undercuts need to be avoided in order to facilitate removing the molded part. Simple superficial cavities ensure a uniform distribution of the mold mass in the tool and a smooth surface of the module resulting in consistent quality of the product [39]. Embedded spacer structures were designed following injection molding restrictions, thus avoiding ramifications or undercuts and too detailed structures.

To find a suitable novel spacer design, the literature was studied and new approaches were implemented. In the literature, results from 2D and 3D simulations as well as from experiments were compared. On the basis of these studies, ten structures were investigated in CFD simulations, from which two final structures were selected (see supporting information). The selection process based on a good agreement between shear rate at the membrane, shear rate distribution, and pressure drop between inlet and outlet. The selected structures, a staggered herringbone and a sinusoidal corrugation, were compared to the empty channel in simulations and additionally to an inlay net spacer in experiments.

Empty channel. The feed channel measures a width of $13.5 \mathrm{~mm}$ and a length of $76 \mathrm{~mm}$. Channel heights on the feed and permeate sides are chosen in accordance with the used spacers. The investigated feed side channel was set to a height of $250 \mu \mathrm{m}$.

Herringbones. Staggered herringbone structures improve mixing in microfluidics and are adapted to this work for different element distances and the element height. To form herringbones, rods of $0.22 \mathrm{~mm}$ radius and $32 \mu \mathrm{m}$ membrane distance are arranged in an angle of $60^{\circ}$ and $3 \mathrm{~mm}$ of distance. The dimensions result in 22 rods along the feed channel.

Sinusoidal corrugation. To create the sinusoidal surface, a basic element with four perpendicular sides is constructed. The adjacent sides of the basic element display sinus functions, which differ by half a period. As the sinus functions are orthogonal, they form a cross-shaped pattern. A complete sinus wave measures $4 \mathrm{~mm}$ and, like the herringbone structure, has 
a membrane distance of $32 \mu \mathrm{m}$. These dimensions result in 19 peaks for the feed channel length. As a variation of the sinusoidal corrugation, the number of peaks is doubled.

\subsection{Fluid dynamics simulation}

To perform the experiments under well-defined flow conditions, the design process of the membrane module was assisted with CFD simulations using COMSOL Multiphysics. In particular, the flow conditions at the membrane fluid interface were optimized with the geometry of the embedded spacers. The three different spacer configurations were simulated with laminar inflow conditions and zero pressure at the outlet. The simulation used pure water from the COMSOL material library for the fluid properties. Geometrical adjustments (e.g. symmetry) and periodic boundary conditions were applied to the simulations to reduce the calculation effort. The mesh was refined, leading to a stable pressure loss probed between the inlet and outlet. Based on the CFD simulation results, the spacer geometry was altered using computer-aided design (CAD) to influence and adapt the desired homogeneous flow field inside the membrane module.

The inlet velocity was set to $0.152 \mathrm{~m} \mathrm{~s}^{-1}$, which equals a flux of $6.7 \mathrm{~mL} \mathrm{~min}^{-1}$ passing the inlet cross-section of $1 \mathrm{~mm}$ diameter. These flow condition results in a Reynolds number of 18 for the empty feed channel. In accordance with Baker [40], simulations can be done for the case of laminar flow.

Navier-Stokes equations for an infinitesimal control volume serve as the basis for evaluating the fluid flow [41]. The conservation of momentum is described by the correlation:

$$
\rho(\boldsymbol{u} \cdot \nabla \boldsymbol{u})=\nabla \cdot\left[-p \boldsymbol{I}+\mu\left(\nabla \boldsymbol{u}+(\nabla \boldsymbol{u})^{T}\right)-\frac{2}{3} \mu(\nabla \cdot \boldsymbol{u}) \boldsymbol{I}\right]+\boldsymbol{F},
$$

where $\rho$ stands for the density, $\boldsymbol{u}$ describes the velocity vector, $p$ is the pressure, $\mu$ is the dynamic viscosity, $\boldsymbol{I}$ is the identity matrix, $\nabla$ is the Nabla operator and $\boldsymbol{F}$ are external forces. In this case, no external forces are applied so $\boldsymbol{F}$ is not accounted. The fluid is assumed to be Newtonian, incompressible and continuous. Thus, the viscous force term $-\frac{2}{3} \mu(\nabla \cdot \boldsymbol{u}) \boldsymbol{I}$ is also neglected. The Navier-Stokes equations are solved together with the continuity equation:

$$
\nabla \cdot \underset{6}{(\rho \boldsymbol{u}})=0
$$


describing the conservation of mass.

Shear stress is a tangential force generated through distortion of a fluid element and reduces the formation of fouling layers on membrane and module surfaces. Thus, high shear rates are considered to increase the permeation rate of a membrane $[22,23,30]$. Since the presented module structures are to be compared on the basis of their permeate flow as proof of performance, the shear rates at the surface are examined. This study compares shear rates averaged over the membrane surface for mentioned embedded spacer structures. The shear rate is a tensor of the strain-rate:

$$
\dot{\gamma}=\left(\nabla \boldsymbol{u}(\nabla \boldsymbol{u})^{T}\right)
$$

Laminar flow indicates further assumptions for this case including settings regarding the time, temperature, solver and initial fluid properties. As no change in time is desired, the flow is investigated under steady-state condition. The model sets the temperature to $293.15 \mathrm{~K}$ and neglects change in temperature. All simulations assume a full developed and constant feed flux. Default selection results in the stationary solver with fully coupled conditions and direct iterations using the PARDISO solver. The mesh system consists of a refinement of boundary layers at the membrane and a total of $10^{5}$ mesh elements for the empty channels.

Concentration polarization plays a significant role in ultrafiltration processes. This work implements transport of diluted species to the model to describe mass transport and thus concentration polarization at the membrane/ fluid interface. To model concentration polarization, fluid-like conditions of dissolved salt in water are introduced.

Extending laminar flow by transport of diluted species merges convective with diffusive flow. This study bases their combination on the following assumptions:

- membrane rejection of $100 \%$ for salt;

- constant ambient pressure and pure water on permeate side;

- constant feed composition with a fixed salt concentration; 
- and density, viscosity and diffusion coefficients independent from concentration. entry concentration, $c_{p}$ for the permeate concentration and $A_{w}$ for the membrane permeability.

Table 1: System conditions for simulation with concentration polarization using material properties according to $[43]$

\begin{tabular}{lll}
\hline Parameter & Value & Unit \\
\hline Laminar flow & & \\
$\mathrm{v}_{\text {in }}$ & 0.152 & $\mathrm{~m} \mathrm{~s}^{-1}$ \\
$\mathrm{p}_{\text {out }}$ & 0 & bar
\end{tabular}

\section{Transport of diluted species}

$\begin{array}{lll}\mathrm{c}_{0} & 6.23 & \mathrm{~mol} \mathrm{~m}^{-3} \\ \mathrm{c}_{\mathrm{p}} & 0 & \mathrm{~mol} \mathrm{~m}^{-3} \\ \mathrm{D} & 1.5 \times 10^{-9} & \mathrm{~m}^{2} \mathrm{~s}^{-1}\end{array}$

\section{General}

$\mathrm{A}_{\mathrm{w}}$

$9.3943 \times 10^{-12} \quad \mathrm{~m}^{2} \mathrm{~s} \mathrm{~kg}^{-1}$

Diluted component Chlorophenol

Transport of diluted species calculates the convective and diffusive movement of a species diluted in water according to equation:

$$
\nabla \cdot\left(-D_{i} \nabla c_{i}\right)+\boldsymbol{u} \cdot \nabla c_{i}=R_{i}
$$

$D$ denotes the diffusion coefficient and $i$ describes the component. As no reaction takes place, the reaction term $R_{i}$ is not assigned. Convective flow characterizes flow through the bulk; diffusive flow describes the laminar boundary layer in the vicinity of the module walls. At 
walls - including the membrane - fluid slows down to $v=0$ and diffusion predominates over convection.

Calculations are complemented by the conservation of mass equation:

$$
\boldsymbol{N}_{i}=-D_{i} \nabla c_{i}+\boldsymbol{u} c_{i}
$$

where $\boldsymbol{N}_{i}$ is the amount of total moles. Boundary conditions remain the same compared to laminar flow, supplemented by additional inflow and outflow for the concentrated species. The extension by transport of diluted species demands a further characterization of the transport at the membrane. The model provides a permeate flux as a second outlet condition as function of the local pressure and concentration at the membrane. The membrane flux increases with higher pressure and decreases with increasing osmotic pressure due to higher local concentration (see equation (6)). In this case, the additional membrane flux is described as the orthogonal outlet velocity $U_{p}$, where the characteristics of the membrane are summarized in the permeability or solvent transport coefficient $A_{w}$ :

$$
U_{p}=A_{w} \cdot(p-(R \cdot T \cdot c))
$$

All other boundaries are set to no-flux condition. According to equation (6), the permeate volume flow depends on pressure $\mathrm{p}$ at the membrane. To enable permeate volume flow, trans-membrane pressures (TMPs) from 2.5 bar to 12.5 bar were applied in the laminar flow node according to

$$
\mathrm{TMP}=\frac{p_{\text {Outlet }}+p_{\text {Inlet }}}{2}-p_{\text {Permeate }}
$$

To compare the different structures, the permeate volume flow was determined by integrating the orthogonal outlet velocity across the membrane surface for the TMPs.

\subsection{Filtration setup and (flow-) MRI measurements}

The technique of magnetic resonance imaging (MRI) allows the visualization of structures and fluid flow inside membrane modules in situ and non-invasively. It utilizes the property of nuclear spins reacting to a magnetic field. 
In this study, the measurements were performed on a Magritek low-filed NMR tomography system. It operates at a Larmor frequency of $23.8 \mathrm{MHz}$ with a field strength of $0.56 \mathrm{~T}$. A 2D spin-echo pulse sequence was used in all measurements. MRI parameters are provided in Table 2. The signal-to-noise ratio in all measurements was larger than 10. The measuring and post-processing technique was done with MATLAB combined with ProsPa (Magritek) and is explained in more detail in our previous studies. [33, 44-47].

Filtration experiments were conducted in a commercially available $30 \mathrm{kDa}$ ambr ${ }^{\circledR}$ crossflow module modified as a screening filter for a broad range of concentrations and molecules. A constant feed flow of $10 \mathrm{~mL} \mathrm{~min}^{-1}$ of a buffer solution consisting of de-ionized water and $1.48 \mathrm{~g} \mathrm{~L}^{-1}$ copper(II) sulfate pentahydrate $\left(\mathrm{CuSO}_{4} \cdot 5 \mathrm{H}_{2} \mathrm{O}\right.$, CAS: 7758-99-8, Carl Roth, Germany) was set using two continuous neMESYS syringe pumps by CETONI GmbH, Germany. A bubble trap was used to prevent air from entering the module. The pressure in the retentate channel was set to 1.5 bar over-pressure using a back-pressure valve.

Table 2: MRI parameter settings for the flow measurement of the ambr ${ }^{\circledR}$ crossflow module.

\begin{tabular}{lll}
\hline & Value & Unit \\
\hline Repetition time & 400 & $\mathrm{~ms}$ \\
Echo time & 32 & $\mathrm{~ms}$ \\
Spatial resolution $[\mathrm{x} \cdot \mathrm{y} \cdot \mathrm{z}]$ & $30 \cdot 8 \cdot 10$ & $\mathrm{~mm} \cdot \mathrm{mm} \cdot \mathrm{mm}$ \\
Number of pixels $[\mathrm{x} \cdot \mathrm{y}]$ & $256 \cdot 128$ & - \\
Number of scans & 12 & - \\
\hline
\end{tabular}

\subsection{Experimental characterization of mass transport}

Simulation results determined the most promising spacer structures and motivated those to be 3D-printed and tested in experiments with protein filtration. The proposed embedded spacer structures were supplemented by and compared with commercial net spacers.

The tested ultrafiltration membrane was a Hydrosart ${ }^{\circledR} 30 \mathrm{kD}$ by Sartorius Stedim $\mathrm{GmbH}$. It was placed either in an empty 3D-printed prototype or in 3D-printed modules with 

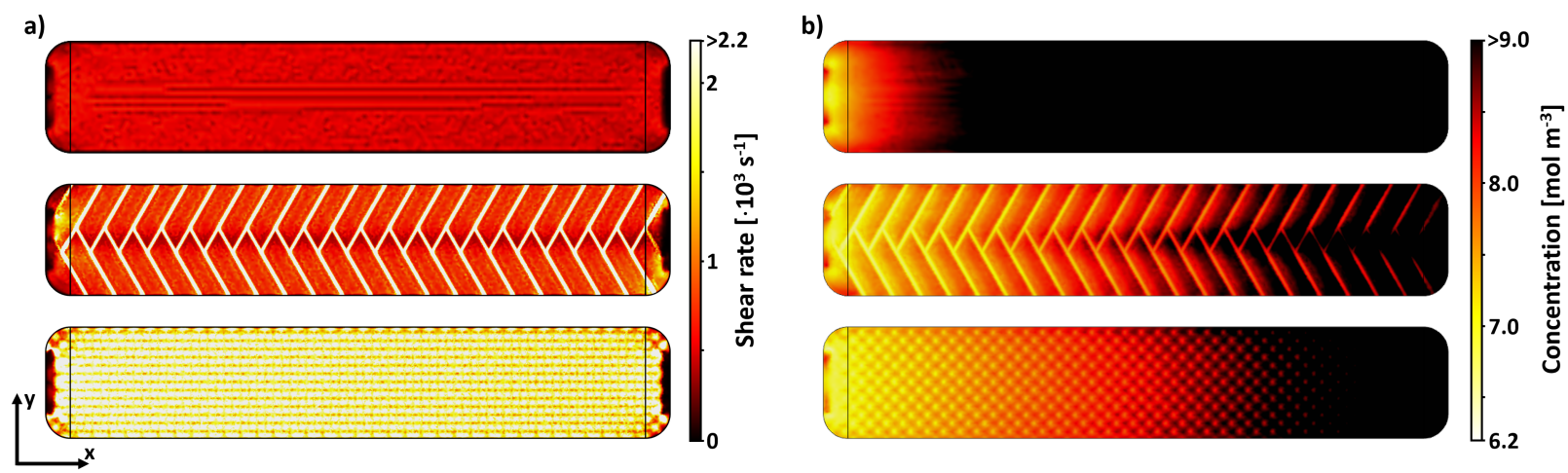

Figure 2: a) CFD simulations show (top) even but low shear rate distribution for the empty channel, (middle) shear rate peaks above the herringbone structures, and (bottom) various peaks and valleys for the sinusoidal corrugation. b) (top) In the empty channel, the concentration increases fast due to concentration polarization. (middle) The herringbone structure delays the concentration increase. (bottom) The Sinus proposes the slowest concentration increase and an even distribution.

embedded structures. In the empty module, a Clearedge $230 \mu \mathrm{m}$ acted as a benchmark mesh-type spacer, which was placed on top of the membrane. Six screws then fixed the module housing at a torque of $1 \mathrm{Nm}$ with a torque wrench (DremoMeter DBGM Rahsol $10-100 \mathrm{~cm} \mathrm{kp})$.

Due to the pre-wetting of the membrane with glycerin, each module was first flushed with $100 \mathrm{~mL}$ ultrapure water. All modules were tested for integrity and water flux beforehand. The water flux test included flushing of $100 \mathrm{~mL}$ solution while tracking the permeate. Only modules with a specific, membrane-related permeate quantity according to the datasheet were used for the experiments. Water flux test and experiments were performed at a back-pressure of 1.5 bar in the retentate while the permeate channel remained open to ambient pressure (retentate outlet was closed for water flux test). The inlet feed volume flow was set to $10 \mathrm{~mL} \mathrm{~min}{ }^{-1}$. The module operated vertically from bottom to top to prevent gas bubble formation. During the experiments, the retentate and feed pressure, the volume flow, and the amount of permeate were recorded. Permeate volume flow was measured simply by using a stopwatch and a measuring cylinder. The syringe pump (PHD Ultra, Harvard Apparatus) ran a $50 \mathrm{ml}$ plastic syringe (Terumo). Pressure sensors (High-Tech EL-PRESS FLOW-BUS, 
Bronkhorst) tracked inlet and outlet pressure. BSA (CAS 9048-46-8, Carl Roth) was used to

prepare a $10 \mathrm{~g} \mathrm{~L}^{-1}$ and a $50 \mathrm{~g} \mathrm{~L}^{-1}$ solution in $10 \mathrm{mmol}$ phosphate buffer of $\mathrm{pH} 7$, pre-filtered with $0.2 \mu \mathrm{m}$ filter.

\section{Results and discussion}

\subsection{Laminar flow simulations visualize hydrodynamics}

Several studies have shown that high shear rates improve mixing and thus mass transfer $[9,30]$. Therefore, this study compares spacer designs to one another in order to find high shear rate distributions averaged over the membrane. CFD results show that the highest shear rates (shown in white in Figure 2 a)) are achieved for small distances between membrane and module wall.

The empty channel (Figure 2 a) (top)) shows an even shear rate distribution with low shear rates around $0.5 \times 10^{3} \mathrm{~s}^{-1}$. For spacer designs, where the shape runs over the whole module width, shear rate peaks appear above the top area of the spacer shape. This is the case for the herringbone structure (Figure 2 a) (middle)), where high shear rates are observed over the diagonal bars with $45 \mu \mathrm{m}$ distance compared to low shear rates (in red) in between those bars (50 $\mathrm{\mu m}$ distance to the membrane).

In the case of the sinusoidal corrugation, the filaments do not form a continuous barrier over the whole width; instead, alternating peaks and valleys appear. The varying structure results in an even distribution and high shear rates (around $2 \times 10^{3} \mathrm{~s}^{-1}$, see Figure 2 a) (bottom)), simultaneously.

\subsection{Salt retention simulations}

Solutes in ultrafiltration processes have sizes of about $0.01-0.5 \mu \mathrm{m}$ and demand particle representations and hydrodynamics to be described by CFD simulations combined with discrete elements representing the particle [48]. However, this emerging simulation technology goes far beyond the questions to be answered here and is too microscopic and hence too simulation-intensive. Instead, we use salt retentions to investigate and represent the effect of flow conditions on solute retention for the different geometries. 
a)

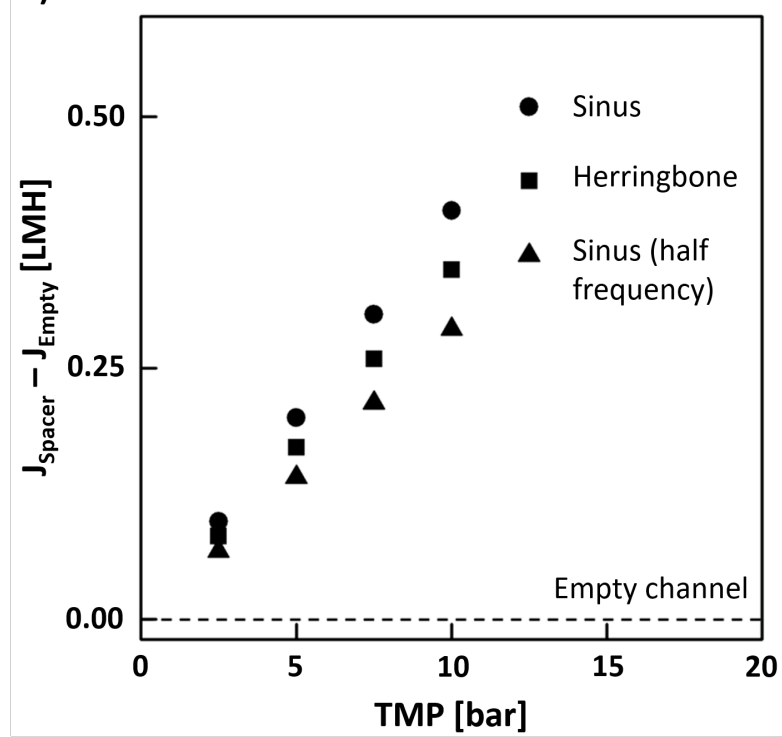

b)

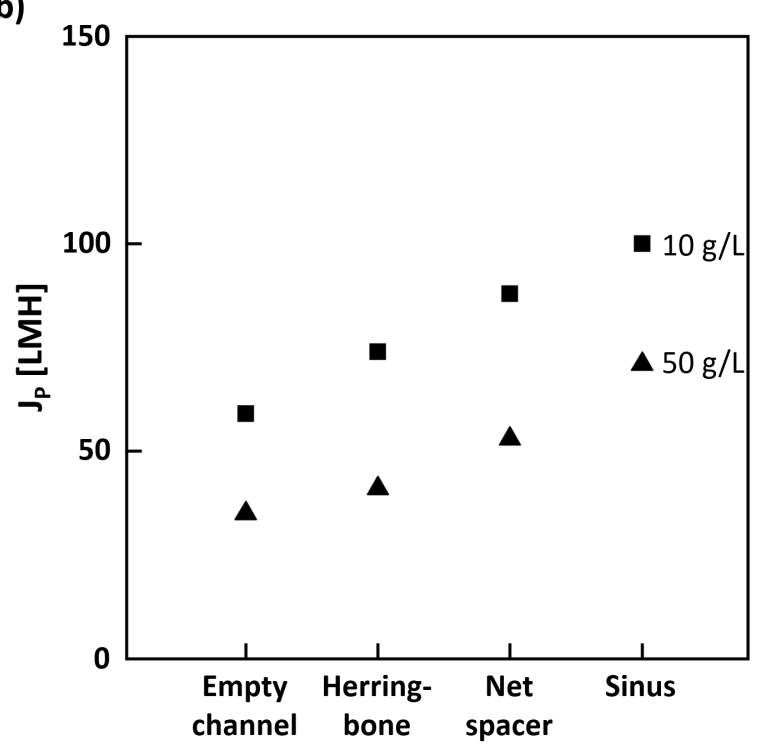

Figure 3: a) Simulations show the highest permeate flux enhancement for the sinusoidal corrugation, followed by herringbone structure and the sinusoidal corrugation with half the frequency. All results are better than those for the empty channel. b) sinusoidal corrugation achieves the highest permeate flux in BSA experiments, followed by the commercial net spacer, herringbone structure, and the empty channel. 
A fluid can be described by continuous movement under tangential or shear force [41]. This is the case for dissolved salts in a diluted solution of water. In section 2.3, fluid-like conditions were introduced to model concentration polarization. To use fluid-like conditions and enable simulation time of few days only, the ultrafiltration system was replaced by a reverse osmosis process. Ultrafiltration and reverse osmosis can be considered similar regarding mechanisms creating concentration polarization [7]. This study uses specifications for a reverse osmosis membrane and its permeability for the concentration of chlorophenol studied by Al-Obaidi and Mujtaba [42]. The calculated results of Al-Obaidi and Mujtaba were validated by experiments [43].

To investigate the correlation between shear rate and concentration, 2D plots for the concentration at the membrane surface are compared. Low concentrations at the membrane are desired as they result in a small osmotic pressure increasing the permeate flux (see the function for the permeate flux in equation (6)). In the legend in Figure $2 \mathrm{~b}$ ), light colors indicate low concentrations. Figure $2 \mathrm{~b}$ ) shows a concentration increase from left to right, which correlates with the feed-flow direction. The concentration trend implies an increase in the number of retained components. The empty channel (Figure $2 \mathrm{~b}$ ) (top)) shows rapid growth in concentration at an early position within the first eighth. The herringbone and the sinusoidal corrugations (Figure $2 \mathrm{~b}$ )(middle) and (bottom)) show that high concentrations are reached at a later point: the herringbone structure reaches the same concentration level as the empty channel at halfway, whereas the sinusoidal corrugation delays the concentration increase up to the last third. As in the case of shear rates, peaks (indicated by light colors) are observed on top of obstacles: above bars for the herringbone structure and above sinus peaks for the sinusoidal corrugation. Where there were high shear rates above the filaments of the herringbone structure in Figure 2 a) (middle), there are now low concentrations in the same regions (lighter colors compared to the space between filaments). The sinusoidal corrugation represents a more even concentration distribution compared to the herringbone structure.

The volume flow of the empty spacer $J_{\text {empty }}$ is compared to the permeate volume flow of the structures $J_{P}$ for different TMPs according to $J_{\text {compare }}=J_{P}-J_{\text {empty }}$ (see Figure 3 a)). 
The permeate volume flow rises for increasing TMP according to equation (6). Figure 3 a) also shows a higher permeate volume flow for the sinusoidal corrugation compared to the herringbone structure. A second sinusoidal corrugation with half the frequency, and thus half the number of sinus peaks, loses performance and shows lower permeate volume flows.

\subsection{Flow-MRI shows mixed fluid flow distribution}

Flow-MRI measurements under constant flow were conducted for the sinusoidal corrugation. Figure 4 a) depicts a magnetic resonance image of the cross-section of the membrane module. The image reveals the inner structure of the membrane module consisting of sixteen permeate channels with a membrane on top and the feed channel with the embedded sinusoidal corrugation. The brighter the color in the magnetic resonance image, the higher the density in the respective pixel in the image. The reason for the fifth permeate channel from the left not being visible is the absence of water in the measuring area. Figure $4 \mathrm{~b}$ ) depicts a flow magnetic resonance measurement of radial velocities inside the membrane module with magnifications in Figure $4 \mathrm{c}$ ). The color bar identifies the velocity magnitude, whereas the arrows represent the direction of flow. Higher velocities of up to $8 \mathrm{~mm} \mathrm{~s}^{-1}$ (dark red) are located at the top of the cross-section at the same position as the sinusoidal corrugation. Thus, the sinusoidal corrugation can be directly correlated with the local velocity increase in radial direction. Furthermore, arrows show a vortex formation in the cross-section and support the assumption of mixed flow in the sinusoidal corrugation. Slow fluid-flow velocities down to $0 \mathrm{~mm} / \mathrm{s}$ are indicated in dark blue. In the lower part of the module corresponding to the permeate channels, no significant radial velocities could be measured.

\subsection{Experimental validation from BSA experiments}

Figure $3 \mathrm{~b}$ ) shows the permeate volume flow $J_{P}$ for the empty channel, the herringbone structure, the sinusoidal corrugation (all 3D printed prototypes), and the commercial net spacer, which was placed in the empty membrane module. Permeate volume flows were conducted for BSA concentrations of $10 \mathrm{~g} \mathrm{~L}^{-1}$ and $50 \mathrm{~g} \mathrm{~L}^{-1}$. Permeate volume flow is higher for low BSA concentrations (see equation (6)). The sinusoidal corrugation shows the best 


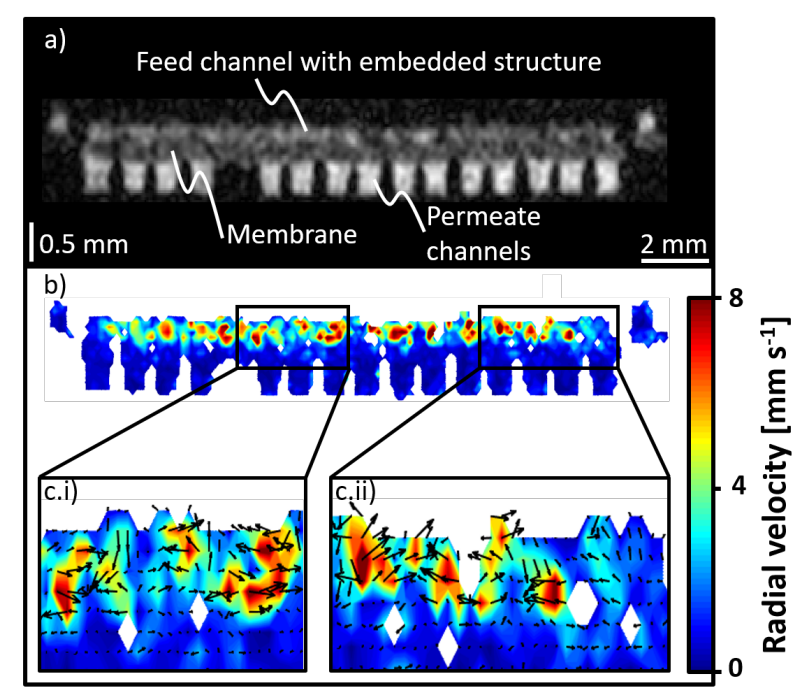

Figure 4: (Flow-)Magnetic resonance imaging on ambr ${ }^{\circledR}$ crossflow module (a) reveals the inner structure of the module, (b) shows high radial velocities, and (c) vortex formation in the sinusoidal structured feed channel.

performance with the highest permeate flow rate, followed by the commercial net spacer, the herringbone structure, and the empty channel. BSA experiments demonstrate that all three spacer structures outperform the empty channel, which is in accordance with literature. Sinusoidal corrugations outperform all others in agreement with the simulations.

\section{Conclusion}

The comprehensive methodology to design fluid compartments with minimized concentration polarization resulted in a novel high-throughput membrane module optimized for flexible use in ultrafiltration screenings.

The chosen structure, the sinusoidal corrugation, shows $10 \%$ to $30 \%$ better results compared to the traditionally used net-shaped spacers. Simulations with a focus on mass transfer and permeate flux visualize that geometrical shape influences velocity gradients and thus shear rate distribution. Simulations show good agreement between shear rate peaks and concentration valleys on the membrane surface and explain the observed permeate flux enhancement. The embedded sinusoidal corrugation outperforms the conventional net-shaped 

concentration profiles, shear sensitive products like cells). 


\section{Acknowledgement}

M.W. acknowledges the support through an Alexander-von-Humboldt Professorship and the European Research Council (ERC) under the European Unions Horizon 2020 research and innovation program (grant agreement no. 694946). This work was also performed in part at the Center for Chemical Polymer Technology CPT, which is supported by the EU and the federal state of North Rhine-Westphalia (grant no. EFRE 3000883 02).

Simulations were performed with computing resources granted by RWTH Aachen University.

\section{References}

[1] G. Belfort, R. H. Davis, A. L. Zydney, The behavior of suspensions and macromolecular solutions in crossflow microfiltration, Journal of Membrane Science 96 (1) (1994) 1 - 58.

[2] R. van Reis, A. Zydney, Bioprocess membrane technology, Journal of Membrane Science 297 (1-2) (2007) $16-50$.

[3] C. Charcosset, Membrane Processes in Biotechnology and Pharmaceutics, Elsevier Science, Burlington, 2012.

[4] H. Lutz (Ed.), Ultrafiltration for bioprocessing: Development and implementation or robusts processes, Vol. 29 of Woodhead Publishing series in biomedicine, Woodhead Publishing, Cambridge, 2015.

[5] G. Belfort, Fluid mechanics in membrane filtration: Recent developments, Journal of Membrane Science 40 (2) (1989) 123-147.

[6] G. B. van den Berg, C. A. Smolders, Flux decline in ultrafiltration processes, Desalination 77 (1990) $101-133$.

[7] E. Matthiasson, B. Sivik, Concentration polarization and fouling, Desalination 35 (1980) 59 - 103.

[8] C. Bhattacharjee, V. K. Saxena, S. Dutta, Static turbulence promoters in cross-flow membrane filtration: a review, Chemical Engineering Communications 207 (3) (2020) 413-433.

[9] A.R. Da Costa, A.G. Fane, D.E. Wiley, Ultrafiltration of whey protein solutions in spacer-filled flat channels, Journal of Membrane Science 76 (1-3) (1993) 245-254.

[10] S. K. Karode, A. Kumar, Flow visualization through spacer filled channels by computational fluid dynamics I.: Pressure drop and shear rate calculations for flat sheet geometry, Journal of Membrane Science 193 (1) (2001) $69-84$.

[11] A. R. Da Costa and A. G. Fane and C. J. D. Fell and A. C. M. Franken, Optimal channel spacer design for ultrafiltration, Journal of Membrane Science 62 (3) (1991) 275 - 291. 
[12] O. Kavianipour, G. D. Ingram, H. B. Vuthaluru, Investigation into the effectiveness of feed spacer configurations for reverse osmosis membrane modules using computational fluid dynamics, Journal of Membrane Science 526 (2017) 156-171.

[13] J. Balster, D. F. Stamatialis, M. Wessling, Membrane with integrated spacer, Journal of Membrane Science 360 (1-2) (2010) 185-189.

[14] I. G. Rácz, J. Wassink, R. Klaassen, Mass transfer, fluid flow and membrane properties in flat and corrugated plate hyperfiltration modules, Desalination 60 (3) (1986) 213-222.

[15] S. Mazinani, A. Al-Shimmery, Y. J. Chew, D. Mattia, 3D Printed Fouling-Resistant Composite Membranes, ACS Applied Materials \& Interfaces 11 (29) (2019) 26373-26383.

[16] H. S. Abid, D. J. Johnson, R. Hashaikeh, N. Hilal, A review of efforts to reduce membrane fouling by control of feed spacer characteristics, Desalination 420 (2017) 384-402.

[17] J.-Y. Lee, W. S. Tan, J. An, C. K. Chua, C. Y. Tang, A. G. Fane, T. H. Chong, The potential to enhance membrane module design with 3D printing technology, Journal of Membrane Science 499 (2016) $480-490$.

[18] R. Kodým, F. Vlasák, D. Šnita, A. Černín, K. Bouzek, Spatially two-dimensional mathematical model of the flow hydrodynamics in a channel filled with a net-like spacer, Journal of Membrane Science 368 (1-2) (2011) 171-183.

[19] F. F. Rivera, L. Fabián, P. Hidalgo, G. Orozco, Study of Hydrodynamics at Asahi ${ }^{\mathrm{TM}}$ prototype electrochemical flow reactor, using computational fluid dynamics and experimental characterization techniques, Electrochimica Acta 245 (2017) 107-117.

[20] S. S. Bucs, R. V. Linares, J. O. Marston, A. I. Radu, J. S. Vrouwenvelder, C. Picioreanu, Experimental and numerical characterization of the water flow in spacer-filled channels of spiral-wound membranes, Water Research 87 (2015) 299 - 310.

[21] A. L. Ahmad, K. K. Lau, M. Z. Abu Bakar, Impact of different spacer filament geometries on concentration polarization control in narrow membrane channel, Journal of Membrane Science 262 (1-2) (2005) 138-152.

[22] Z. Cao, D.E. Wiley, A.G. Fane, CFD simulations of net-type turbulence promoters in a narrow channel, Journal of Membrane Science 185 (2) (2001) 157-176.

[23] Y. K. Lee, Y.-J. Won, J. H. Yoo, K. H. Ahn, C.-H. Lee, Flow analysis and fouling on the patterned membrane surface, Journal of Membrane Science 427 (2013) 320-325.

[24] J. E. Lira-Teco, F. Rivera, O. Farías-Moguel, J. Torres-González, Y. Reyes, R. Antaño-López, G. Orozco, F. Castañeda-Zaldivar, Comparison of experimental and CFD mass transfer coefficient of three commercial turbulence promoters, Fuel 167 (2016) 337-346.

[25] J. Liu, A. Iranshahi, Y. Lou, G. Lipscomb, Static mixing spacers for spiral wound modules, Journal of Membrane Science 442 (2013) 140-148. 
[26] C. Rodrigues, V. Geraldes, M. N. de Pinho, V. Semião, Mass-transfer entrance effects in narrow rectangular channels with ribbed walls or mesh-type spacers, Chemical Engineering Science 78 (2012) $38-45$.

[27] J. Schwinge, D. E. Wiley, A. G. Fane, R. Guenther, Characterization of a zigzag spacer for ultrafiltration, Journal of Membrane Science 172 (1-2) (2000) 19-31.

[28] S. Y. Jung, J. E. Park, T. G. Kang, K. H. Ahn, Design Optimization for a Microfluidic Crossflow Filtration System Incorporating a Micromixer, Micromachines 10 (12) (2019) 836.

[29] A. Shrivastava, S. Kumar, E. L. Cussler, Predicting the effect of membrane spacers on mass transfer, Journal of Membrane Science 323 (2) (2008) 247-256.

[30] C. P. Koutsou, S. G. Yiantsios, A. J. Karabelas, Numerical simulation of the flow in a plane-channel containing a periodic array of cylindrical turbulence promoters, Journal of Membrane Science 231 (1-2) (2004) 81-90.

[31] G. Schock, A. Miquel, Mass transfer and pressure loss in spiral wound modules, Desalination 64 (1987) $339-352$.

[32] J. Balster, I. Pünt, D. Stamatialis, M. Wessling, Multi-layer spacer geometries with improved mass transport, Journal of Membrane Science 282 (1-2) (2006) 351 - 361.

[33] M. Wiese, S. Benders, B. Blümich, M. Wessling, 3D MRI velocimetry of non-transparent 3D-printed staggered herringbone mixers, Chemical Engineering Journal 343 (2018) 54-60.

[34] C. Fritzmann, M. Hausmann, M. Wiese, M. Wessling, T. Melin, Microstructured spacers for submerged membrane filtration systems, Journal of Membrane Science 446 (2013) 189 - 200

[35] C. Fritzmann, M. Wiese, T. Melin, M. Wessling, Helically microstructured spacers improve mass transfer and fractionation selectivity in ultrafiltration, Journal of Membrane Science 463 (2014) 41 - 48.

[36] S. Armbruster, O. Cheong, J. Lölsberg, S. Popovic, S. Yüce, M. Wessling, Fouling mitigation in tubular membranes by 3D-printed turbulence promoters, Journal of Membrane Science 554 (2018) 156 - 163.

[37] S. Popović, M. Wessling, Turbulence Promoters in Membrane Processes, Journal of Membrane Science Virtual Issue, 2015.

[38] T. Femmer, I. Flack, M. Wessling, Additive Manufacturing in Fluid Process Engineering, Chemie Ingenieur Technik 88 (5) (2016) 535-552.

[39] I. Gibson, D. Rosen, B. Stucker, Additive manufacturing technologies: 3D printing, rapid prototyping and direct digital manufacturing, 2nd Edition, Springer, 2015.

[40] R. W. Baker, Membrane Technology and Applications, 3rd Edition, John Wiley \& Sons, 2012.

[41] N. Datta, B. K. Guha, Mass transfer limitations in a membrane module, Desalination 89 (3) (1993) $343-358$.

[42] M. A. Al-Obaidi, I. M. Mujtaba, Steady state and dynamic modeling of spiral wound wastewater reverse 
osmosis process, Computers \& Chemical Engineering 90 (2016) 278-299.

[43] S. Sundaramoorthy, G. Srinivasan, D. Murthy, An analytical model for spiral wound reverse osmosis membrane modules: Part II — Experimental validation, Desalination 277 (1-3) (2011) 257-264.

[44] D. Wypysek, D. Rall, M. Wiese, T. Neef, G.-H. Koops, M. Wessling, Shell and lumen side flow and pressure communication during permeation and filtration in a multibore polymer membrane module, Journal of Membrane Science 584 (8) (2019) 254-267.

[45] T. Luelf, D. Rall, D. Wypysek, M. Wiese, T. Femmer, C. Bremer, J. U. Michaelis, M. Wessling, 3D-printed rotating spinnerets create membranes with a twist, Journal of Membrane Science 555 (2018) $7-19$.

[46] M. Wiese, C. Malkomes, B. Krause, M. Wessling, Flow and filtration imaging of single use sterile membrane filters, Journal of Membrane Science 552 (2018) 274-285.

[47] M. Wiese, O. Nir, D. Wypysek, L. Pokern, M. Wessling, Fouling minimization at membranes having a 3D surface topology with microgels as soft model colloids, Journal of Membrane Science 569 (2019) 7-16.

[48] J. Lohaus, Y. M. Perez, M. Wessling, What are the microscopic events of colloidal membrane fouling?, Journal of Membrane Science 553 (2018) 90-98. 